\section{More on the Resolution of Bisphosphonate-associated Osteonecrosis of the Jaw}

\section{To the Editor:}

We had one case similar to that reported by Lau, et $a l^{1}$. The patient is a 72-year-old woman who sustained a painful fistula (Figure 1a) after dental implants 6 months before. She had taken alendronate weekly for 4 years. She underwent sequestrectomy of necrotic bone, oral antibiotics, and oral irrigation with aqueous iodine solution. After 5 months of therapy, the situation deteriorated (Figure 1b). She was started on anabolic therapy with teriparatide $(20 \mu \mathrm{g})$ in May 2006. After 5 months of teriparatide, significant bone regeneration was found and the mandibular fistula was healing (Figures 1c and 1d). At 10 months' followup, the panoramic radiograph showed complete resolution of the necrotic region (Figure 2).

Bisphosphonates play an important role in osteoporosis management. Even if the rate is very $l w^{2}$, bisphosphonate-related osteonecrosis of the jaw (BRONJ) produces significant morbidity. Prevention and treatment strategies are currently based on expert opinion and focus on maintaining good oral hygiene and conservative surgical intervention ${ }^{3}$.

Teriparatide is the only available anabolic agent for managing osteoporosis. It also shows potential for enhancing fracture healing ${ }^{4}$. Currently, there is no standard for treating BRONJ; we share our experience with satisfactory results after using teriparatide, which might be a promising solution for $\mathrm{ONJ}$.
KUO-YANG TSAI, DDS; CHIN-SHENG HUANG, DDS; GUAN-MIN HUANG, DDS, Department of Oral and Maxillofacial Surgery;

CHEN-TUNG YU, MD, MSc, Chief, Section of Bone Disease, Department of Orthopedic Surgery, Changhua Christian Hospital, 135 Nan-shiao Street, Changhua City 500, Taiwan. Address correspondence to Dr. Yu; E-mail: ortho.yu@msa.hinet.net

\section{REFERENCES}

1. Lau AN, Adachi JD. Resolution of osteonecrosis of the jaw after teriparatide [recombinant human PTH-(1-34)] therapy. J Rheumatol 2009;36:1835-7.

2. Cartsos VM, Zhu S, Zavras AI. Bisphosphonate use and the risk of adverse jaw outcomes: a medical claims study of 714,217 people. J Am Dent Assoc 2008;139:23-30.

3. Khan AA, Sandor GK, Dore E, Morrison AD, Alsahli M, Amin F, et al. Bisphosphonate associated osteonecrosis of the jaw. J Rheumatol 2009;36:478-90.

4. Yu CT, Wu JK, Chang CC, Chen CL, Wei JC. Early callus formation in human hip fracture treated with internal fixation and teriparatide. J Rheumatol 2008;35:2082-3.

J Rheumatol 2010;37:3; doi:10.3899/jrheum.091022
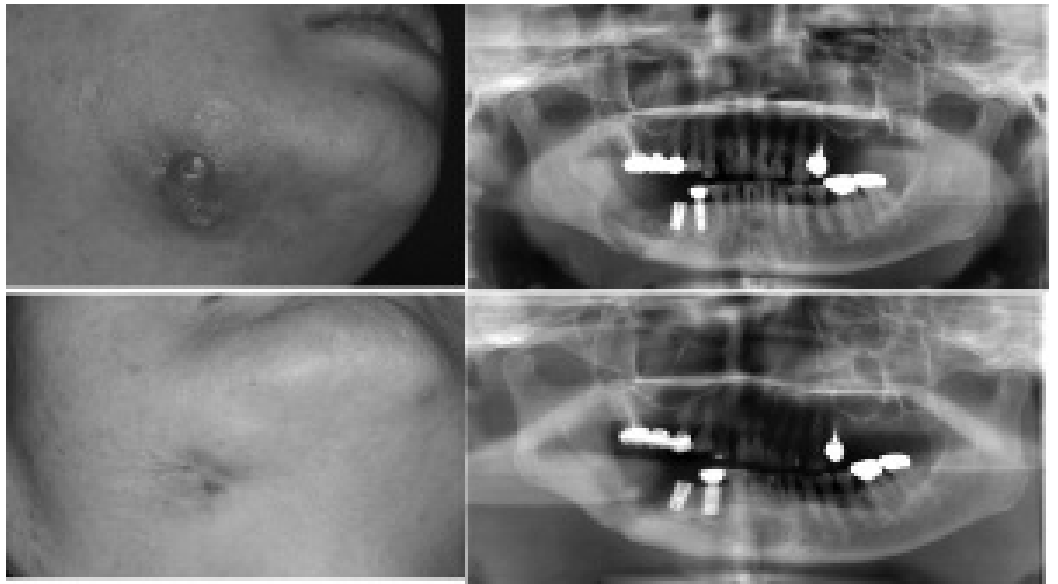

Figure 1. (a) Painful cutaneous fistula with suppuration on right chin. (b) Panoramic radiograph taken 4 months after dental extraction shows an area of alveolar necrosis, apparently localized to the right mandibular areas (arrow). (c and d) The fistula healed with scar formation, and significant bone regeneration was noted after 5 months of therapy.

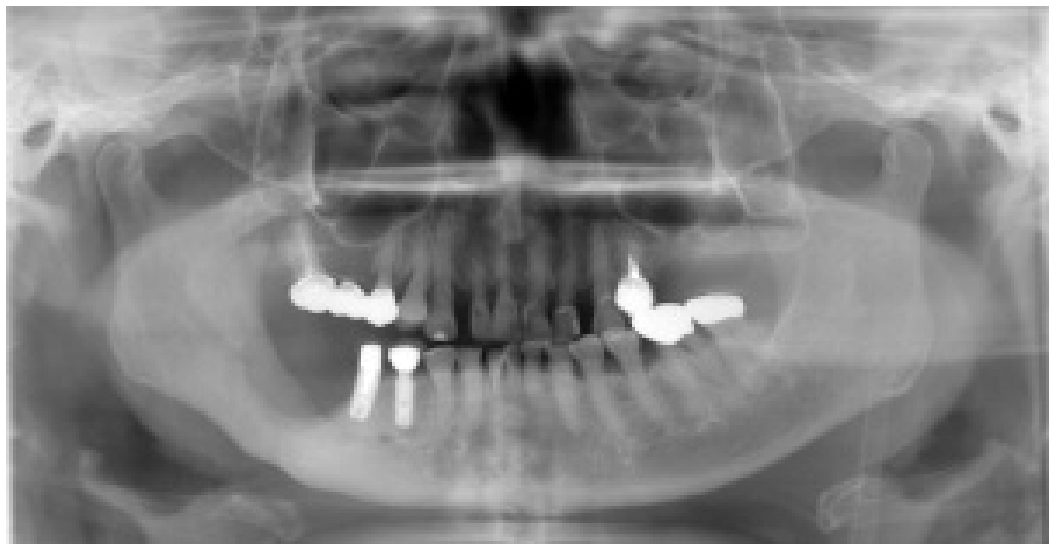

Figure 2. Panoramic radiograph showed complete resolution of necrotic region after 10 months of therapy. 